\title{
Toward Resolving the Reflective-Formative Measurement Debate: Theoretical Framework and an Empirical Investigation-An Abstract
}

\author{
James Agarwal and Oleksiy Osiyevskyy
}

\begin{abstract}
Acknowledging the ontological and epistemological differences inherent between formative and reflective measurement paradigms, we advocate an information processing approach in explaining the efficacy of these approaches in conceptualizing attitudinal constructs. Specifically, we focus on the measurement of corporate reputation, examining two competing measurement conceptualizations: second-order reflective model versus second-order formative model. Drawing from dual-processing theories we explain the underlying theoretical mechanisms at play in the activation of reflective versus formative conceptualizations of corporate reputation contingent upon an individual's level of "need for cognition" trait. Using mobile phone customers in two countries, we provide empirical validation to both conceptualizations, and thus both reflective and formative models are plausible. However, their model fit varies across the levels of individuals" "need for cognition." As a methodological contribution, we propose a novel "jackknifing" methodology in structural equation modeling to conduct statistical tests of our proposed cognitive contingency model. By this means, the study brings us one step closer in an attempt to reconcile the validity of both measurement paradigms.
\end{abstract}

References available upon request.

\footnotetext{
J. Agarwal ( $\square)$

University of Calgary, Calgary, AB, Canada

e-mail: james.agarwal@haskayne.ucalgary.ca

O. Osiyevskyy

Northeastern University, Boston, MA, USA

e-mail: o.osiyevskyy@neu.edu
} 